\title{
Controlled release hydrogen sulfide delivery system based on mesoporous silica nanoparticles protects graft endothelium from ischemia-reperfusion injury
}

This article was published in the following Dove Press journal:

International Journal of Nanomedicine

18 July 2016

Number of times this article has been viewed

\author{
Wenshuo Wangl,* \\ Xiaotian Sun ${ }^{1,2, *}$ \\ Huili Zhang ${ }^{3}$ \\ Cheng Yang' \\ Ye Liu ${ }^{4,5}$ \\ Wuli Yang ${ }^{4,5}$ \\ Changfa Guo' \\ Chunsheng Wang' \\ 'Department of Cardiac Surgery, \\ Shanghai Institute of Cardiovascular \\ Diseases, Zhongshan Hospital, \\ ${ }^{2}$ Department of Cardiothoracic \\ Surgery, Huashan Hospital, Fudan \\ University, ${ }^{3}$ Department of Cardiology, \\ Shanghai Ninth People's Hospital, \\ Shanghai Jiao Tong University, \\ ${ }^{4}$ State Key Laboratory of Molecular \\ Engineering of Polymers, ${ }^{5}$ Department \\ of Macromolecular Science, Fudan \\ University, Shanghai, People's Republic \\ of China \\ *These authors contributed equally \\ to this work
}

\begin{abstract}
Hydrogen sulfide $\left(\mathrm{H}_{2} \mathrm{~S}\right)$ functions as a protective gas transmitter in various physiological and pathological processes, but the lack of ideal donors severely hampers the clinical application of $\mathrm{H}_{2} \mathrm{~S}$. This study aims to construct a controlled release $\mathrm{H}_{2} \mathrm{~S}$ donor and evaluate its protective effect on graft endothelium. Mesoporous silica nanoparticles (MSNs) were synthesized using the sol-gel method and loaded with diallyl trisulfide (DATS), an $\mathrm{H}_{2} \mathrm{~S}$ releasing agent named DATS-MSN. In vitro experiments showed that DATS-MSN could alleviate endothelial cell inflammation and enhance endothelial cell proliferation and migration. In vivo experiments demonstrated that the apoptosis of graft endothelium was mitigated in the presence of DATS-MSN. Our results indicated that DATS-MSN, releasing $\mathrm{H}_{2} \mathrm{~S}$ in a controlled release fashion, could serve as an ideal $\mathrm{H}_{2} \mathrm{~S}$ donor.
\end{abstract}

Keywords: inflammatory response, rejection, cellular uptake, proliferation, cardiac allograft vasculopathy

\section{Introduction}

Cardiac allograft vasculopathy (CAV) is the leading cause of death in heart transplant patients within 1 year of transplant surgery. ${ }^{1}$ The development of CAV is primarily associated with extensive and irreparable damage of endothelial cells (ECs) forming the intima of coronary arteries as a result of ischemia-reperfusion (IR) during the harvest of donor hearts and/or surgical implantation. To address this problem, various preservation strategies have been proposed and implemented, such as maintaining donor hearts at low temperature, maintaining ionic balance to prevent tissue swelling and acidosis, and adding exogenous adenosine triphosphate to preservation solutions. However, donor hearts preserved under these conditions are surgically viable only for an extremely short period of time that often fails to meet the needs of recipients. Therefore, it is important to develop more effective organ preservation methods that can significantly extend the preservation time of donor hearts and ultimately increase the success rate of transplant surgeries.

Recently, hydrogen sulfide $\left(\mathrm{H}_{2} \mathrm{~S}\right)$ has emerged as a new organ-preserving agent in the field of transplantation. ${ }^{2,3} \mathrm{H}_{2} \mathrm{~S}$ has been reported to significantly improve short- and long-term graft function and survival when used as an additive in conjunction with common organ preservation solutions, such as histidine-tryptophan-ketoglutarate solution and the University of Wisconsin (UW) solution. ${ }^{4}$ Despite these benefits, clinical utility of $\mathrm{H}_{2} \mathrm{~S}$ is severely limited by the cytotoxic effects that it demonstrates at high dosages, particularly on mitochondrial membrane transport. ${ }^{5}$ Furthermore, the rate of 
$\mathrm{H}_{2} \mathrm{~S}$ generation is also critical. Depending on the release rate, $\mathrm{H}_{2} \mathrm{~S}$ can affect different biochemical pathways and can elicit drastically varied, even opposing, cellular responses. ${ }^{6,7}$

In the present study, we report a new delivery system based on diallyl trisulfide-loaded mesoporous silica nanoparticles (DATS-MSN) for the controlled release of $\mathrm{H}_{2} \mathrm{~S}$. We also present compelling experimental evidence based on both in vitro cell-based assays and mouse models to elucidate the cytoprotective properties of the nanoparticles and to shed light on the mechanism that underlies their beneficial effects on aortic allografts.

\section{Materials and methods}

\section{Synthesis and characterization of DATS-MSNs}

The synthesis of MSNs was performed using the sol-gel method. ${ }^{8}$ Briefly, $2.1 \mathrm{~mL}$ of $\mathrm{NaOH}$ (2 M; Sigma-Aldrich Co., St Louis, MO, USA) and $0.6 \mathrm{~g}$ of cetyltrimethyl ammonium bromide (99\%; Sigma-Aldrich Co.) were mixed in $300 \mathrm{~mL}$ of deionized water. The mixture was heated to $80^{\circ} \mathrm{C}$ and stirred at $200 \mathrm{rpm}$ for 1 hour, followed by addition of $3 \mathrm{~mL}$ of TEOS (98\%; Sigma-Aldrich Co.). After another 2 hours, the precipitate was collected by centrifugation and washed with water and ethanol twice each. The pore-generating template, cetyltrimethyl ammonium bromide, was then removed by stirring for 2 hours at $80^{\circ} \mathrm{C}$ in $100 \mathrm{~mL}$ of ethanol (99\%; Sigma-Aldrich Co.) containing 1\% (v/v) ammonium hydroxide (99\%; SigmaAldrich Co.). The resultant mixture was washed again with water and ethanol several times each and then vacuum-dried until it assumed the appearance of a white powder.

Drug loading was conducted according to a previously described protocol with minor modifications. ${ }^{9}$ Specifically, $10 \mathrm{mg}$ of MSNs and $8 \mathrm{mg}$ of DATS (Sigma-Aldrich Co.) were sequentially dispersed in $2 \mathrm{~mL}$ of distilled water to afford a mixture with a drug-to-carrier ratio of $0.8: 1$. The mixture was subsequently stirred at room temperature for 12 hours and centrifuged to collect the DATS-loaded composite nanoparticles. Finally, DATS was removed from the surface of MSNs by washing with distilled water. The resultant DATS-MSNs were then dispersed in water to a final concentration of $150 \mathrm{mg} / \mathrm{L}$, and a drop of the suspension was added onto a 200-mesh carbon-coated copper grid. Approximately 5 minutes following the deposition, the water on the grid surface was gently removed by filter paper. The DATS-MSNs on the grid were then air-dried and structurally analyzed using a transmission electric microscope (CM200, Philips, Amsterdam, the Netherlands) at an acceleration voltage of $120 \mathrm{kV}$.

\section{Time course of $\mathrm{H}_{2} \mathrm{~S}$ release}

Time course experiments for $\mathrm{H}_{2} \mathrm{~S}$ release were performed on $\mathrm{H}_{2} \mathrm{~S}$-selective microelectrodes using $10 \mathrm{mg} / \mathrm{L}, 20 \mathrm{mg} / \mathrm{L}$, and $30 \mathrm{mg} / \mathrm{L}$ of DATS-MSNs. The loaded nanoparticles were added to a glass chamber (World Precision Instruments, Sarasota, FL, USA) containing $10 \mathrm{~mL}$ of $2 \mathrm{mM}$ glutathione (GSH; Sigma-Aldrich Co.) in UW solution at $4^{\circ} \mathrm{C} . \mathrm{H}_{2} \mathrm{~S}$ formation was detected using an ISO- $\mathrm{H}_{2} \mathrm{~S}-2$ sensor (World Precision Instruments) attached to an Apollo 1100 Free Radical Analyzer (World Precision Instruments). The concentration of $\mathrm{H}_{2} \mathrm{~S}$ is proportional to the amount of electric current generated on the $\mathrm{H}_{2} \mathrm{~S}$-selective microelectrode. Based on this principle, the level of $\mathrm{H}_{2} \mathrm{~S}$ released from DATS-MSNs was calculated based on the calibration curve constructed from a series of standard NaHS solutions.

\section{Cell culture}

All experimental procedures were approved and performed according to the guidelines of the Institutional Animal Care and Use Committee of Fudan University. ECs were isolated from murine aortas. In brief, mice were sacrificed and aortas were harvested. After ligation of aortic branches, the aortas were rinsed with phosphate-buffered saline (PBS; Beyotime, Nantong, Jiangsu, People's Republic of China) and perfused in F12 medium containing $0.1 \%$ collagenase type II (SigmaAldrich Co.), followed by 15 -minute digestion in a shaker at $60 \mathrm{rpm}$ and $37^{\circ} \mathrm{C}$. The resulting cell suspension was transferred into a $1 \mathrm{~mL}$ sterile conical tube and centrifuged at $1,000 \mathrm{rpm}$ for 10 minutes. After discarding the supernatant, cells were resuspended and cultured in Endothelial Cell Medium (ECM; Sciencell, Carlsbad, CA, USA) to selectively enrich ECs until the fourth or fifth passage.

The identity of the harvested ECs was confirmed by immunofluorescence double staining with antimouse vWF antibody (Abcam, Cambridge, UK) and antimouse CD31 antibody (Miltenyi Biotec GmbH, Bergisch Gladbach, Germany). The cells were further evaluated for lipid uptake using Dil-ac-LDL (Thermo Fisher Scientific, Waltham, MA, USA) and FITC-UEA-I (Sigma-Aldrich, St Louis, MS, USA). The purity of the ECs was determined using a FACSArial flow cytometer (Becton Dickinson, Franklin Lakes, NJ, USA) using the phycoerythrin (PE)-conjugated antihuman CD31 antibody (eBioscience, San Diego, CA, USA).

\section{Uptake assessment}

ECs were cultivated in culture dishes containing $50 \mu \mathrm{g} / \mathrm{mL}$ FITC-conjugated DATS-MSNs in ECM under ambient conditions for 4 hours. The medium was then removed, and 
the cells were washed with PBS twice. After the addition of fresh complete medium, the cells were imaged under a confocal microscope (FV1200; Olympus Corporation, Tokyo, Japan).

\section{Hypoxia-reoxygenation model}

For hypoxia-reoxygenation treatment, $100 \mu \mathrm{L}$ of ECM containing roughly $10^{3}$ ECs was added to each well of a 96-well microelectrode plate and incubated for 24 hours at $37^{\circ} \mathrm{C}$. The medium was then replaced by precooled UW solution containing $2 \mathrm{mM} \mathrm{GSH}$, followed by treatment with different concentrations of DATS-MSNs ranging from $3.13 \mu \mathrm{g} / \mathrm{mL}$ to $800 \mu \mathrm{g} / \mathrm{mL}$ under hypoxic conditions $\left(95 \% \mathrm{~N}_{2} / 5 \% \mathrm{CO}_{2}\right)$ at $4{ }^{\circ} \mathrm{C}$ for 60 minutes. A control was included in which no DATS-MSNs were added. Following the treatment, the UW solution was replaced with an equal volume of ECM, and reoxygenation was performed. The migratory and proliferative properties of ECs subjected to hypoxia-reoxygenation conditions were monitored using the xCELLigence RTCA DP station (ACEA, San Diego, CA, USA), which evaluates cellular events in real time by measuring cell-induced electrical impedance on the microelectrodes.

\section{Tube formation assay}

The group of ECs showing the highest cell index curve were recovered from the DP station and submitted to tube formation assay. In short, Matrigel (Becton Dickinson) was thawed, added to each well of a prechilled sterile 96-well plate, and then allowed to solidify by incubating at $37^{\circ} \mathrm{C}$ for 1 hour. Meanwhile, ECs suspended in ECM at a density of $2 \times 10^{5}$ cells $/ \mathrm{mL}$ were added onto the Matrigel and incubated at $37^{\circ} \mathrm{C}$ for 18 hours. The extent of tube formation was estimated under a light microscope by observing the overall tube lengths and the number of branch points.

\section{Flow cytometric analysis}

To evaluate the effect of DATS-MSNs on endothelial inflammation, ECs were treated with the concentration of DATS-MSNs that produced the highest cell index based on the hypoxia-reoxygenation model, followed by incubation with intercellular adhesion molecule-1-PE (ICAM1-PE) antibody or vascular cell adhesion molecule-1-PE (VCAM-1-PE) antibody (eBioscience) at $4^{\circ} \mathrm{C}$ for 0.5 hours. The cells were then washed twice with PBS containing $2 \%$ bovine serum albumin (Beyotime) and twice again with $0.1 \% \mathrm{NaN}_{3}$. For isotype control, PE-conjugated mouse anti-IgG1 antibody (eBioscience) was used. Subsequently, the cells were resuspended in $500 \mu \mathrm{L}$ of PBS supplemented with $1 \%$ paraformaldehyde (Beyotime) and immediately analyzed using a FACSArial flow cytometer. After correcting nonspecific binding, the percentage of positive cells was determined using FlowJo.

\section{Measurement of mitochondrial membrane potential}

Mitochondrial membrane potential was measured using the MitoCapture Mitochondrial Apoptosis Detection Kit according to the manufacturer's protocol (BioVision, Shinjuku, Tokyo, Japan). MitoCapture is a cationic dye that exhibits different localization patterns depending on the transmembrane potential of mitochondria. The dye selectively aggregates in the mitochondria of healthy cells and emits bright red fluorescence. The altered mitochondrial transmembrane potential of apoptotic cells, in contrast, would cause the dye to remain in the cytoplasm as a greenexcitable fluorescent monomer. Briefly, ECs were suspended in $1 \mathrm{~mL}$ incubation buffer containing $1 \mu \mathrm{L}$ of MitoCapture and incubated for 15 minutes at $37^{\circ} \mathrm{C}$ under $5 \% \mathrm{CO}_{2}$. After staining, the fluorescent signals were measured using a FACSArial flow cytometer. Aggregated MitoCapture was excited at $530 \mathrm{~nm}$, and the red fluorescence that it emitted was detected at $630 \mathrm{~nm}$, whereas the monomeric form of the dye was excited at $488 \mathrm{~nm}$, and the resulting green fluorescence was detected at $530 \mathrm{~nm}$. Mitochondrial dysfunction was indicated by the ratio of red-to-green fluorescence in the generated dot plots.

\section{Animal model}

Balb/c mice aged 4-6 weeks were purchased from Shanghai Laboratory Animal Company (Shanghai, People's Republic of China). Donors and recipients shared the same genetic background in order to avoid host immune rejection. All animals were maintained in the Zhongshan Hospital mouse facilities and allowed free access to food and water.

Transplant surgery was conducted based on a previously described protocol. ${ }^{10}$ Briefly, all mice were anesthetized with pentobarbital (Sigma-Aldrich Co.) at a dosage of $30 \mathrm{mg} / \mathrm{kg}$. A segment of abdominal aorta was harvested from each donor and preserved in chilled UW solution supplemented with the optimal concentration of DATS-MSNs determined in the hypoxia-reoxygenation experiment. UW solution without DATS-MSNs was used as control. Laparotomy was then performed on the recipient mice, in which the section of abdominal aorta between the renal arteries and the iliac bifurcation was ligated. The aortic segment excised from each donor was then anastomosed to a recipient's abdominal 
aorta in an end-to-end fashion. Three days after the surgery, all recipient mice were sacrificed and the transplanted aortic segments were harvested.

\section{Transferase dUTP nick-end labeling assay}

Cryosections of transplanted aortic segments were fixed with $4 \%$ paraformaldehyde for 30 minutes. A terminal deoxynucleotidyl transferase dUTP nick-end labeling (TUNEL) assay was performed to evaluate cell apoptosis in aortic endothelium using the In Situ Cell Death Detection Kit (Hoffman-La Roche Ltd., Basel, Switzerland) and color-developed with 3,3'-diaminobenzidine substrate (Hoffman-La Roche Ltd.) according to the manufacturer's instructions. Apoptotic cells were counted under a light microscope (BX51; Olympus Corporation) aided by cellSens software (Olympus Corporation).

\section{Data analysis}

All data are derived from the mean of duplicate samples from three independently performed experiments and expressed in the format of mean \pm SEM. Statistical analysis was performed using SPSS (Version 20.0; IBM Corporation, Armonk, NY, USA) via either analysis of variance or Student-NewmannKeuls multiple comparison test. Differences were considered statistically significant at $P<0.05$.

\section{Results DATS-MSNs released $\mathrm{H}_{2} \mathrm{~S}$ in a controlled fashion}

Transmission electron microscopy imaging showed that DATSMSNs existed in monodisperse form featuring a narrow size distribution and significant mesoporosity, which could provide a high specific surface area (Figure 1A) and consequently increase the drug payload. The average diameter of DATS-MSNs was estimated to be $\sim 175 \pm 35 \mathrm{~nm}$ (Figure 1B). The time course for $\mathrm{H}_{2} \mathrm{~S}$ release was monitored on an $\mathrm{H}_{2} \mathrm{~S}$-selective microelectrode (Figure 1C). The amount of $\mathrm{H}_{2} \mathrm{~S}$ was proportional to the current generated from the oxidation of $\mathrm{S}^{2-}$ on the surface of the electrode. Based on this principle, we found that the addition of DATS-MSNs to UW solution resulted in the immediate formation and release of $\mathrm{H}_{2} \mathrm{~S}$. The DTAS that existed in the pores reacted with the glutathione in UW solution (Figure 1D). The level of $\mathrm{H}_{2} \mathrm{~S}$ steadily increased to its peak value in $\sim 1$ hour and remained largely stable for an additional period of 3 hours before gradually decreasing. These results suggested that the use of DATS-MSNs could mimic the biological effect of endogenous $\mathrm{H}_{2} \mathrm{~S}$ by achieving controlled delivery of the cytoprotective gas agent with a sustained and steady release profile.

\section{Uptake of DATS-MSNs by ECs}

The quality and purity of the isolated mouse aortic ECs was determined by immunofluorescence staining (Figure 2A-G) and flow cytometric analysis (Figure 2H). FITC-conjugated DATS-MSNs were shown to be able to penetrate through cell membrane and undergo internalization after being incubated with the ECs (Figure 2I-K).

\section{DATS-MSN enhanced the proliferation and differentiation of ECs}

Hypoxia-reoxygenation experiments indicated that ECs treated with $3.13 \mu \mathrm{g} / \mathrm{mL}$ or $6.25 \mu \mathrm{g} / \mathrm{mL}$ of DATS-MSNs showed an increased proliferative capacity, with the former dosage producing the best cytoprotective effect in the first 48 hours (Figure 3A). However, increasing the concentration beyond $6.25 \mu \mathrm{g} / \mathrm{mL}$ abolished these benefits and conversely aggravated EC injury induced by hypoxia-reoxygenation (Figure $3 \mathrm{~A}$ ), possibly due to $\mathrm{H}_{2} \mathrm{~S}$ overdose.

The differentiation capability of ECs was evaluated by tube formation assay. As described earlier, ECs incubated with $3.13 \mu \mathrm{g} / \mathrm{mL}$ of DATS-MSNs (Figure 3B) showed markedly better differentiation and tube formation efficiency compared with the untreated control (Figure 3C), which remained largely unattached from each other. These observations suggested that DATS-MSNs could improve the ability of ECs to proliferate and form vascular structures.

\section{DATS-MSN alleviated inflammatory response through preserving mitochondrial potential}

Inflammatory response of ECs to hypoxia-reoxygenation treatment was characterized by measuring the expression levels of ICAM-1 and VCAM-1 via flow cytometry. Both ICAM-1 and VCAM-1 were shown to be substantially downregulated in cells incubated with $3.13 \mu \mathrm{g} / \mathrm{mL}$ of DATS-MSNs under hypoxic conditions, compared to the untreated control (Figure 4A, ICAM-1, $43.90 \% \pm 6.363 \%$ for treatment group and $18.67 \% \pm 0.688 \%$ for control group, $P<0.05$; Figure 4B, VCAM-1, $15.93 \% \pm 1.828 \%$ for treatment group and $41.77 \% \pm 1.392 \%$ for control group, $P<0.001$; the relevant statistics are shown in Figure 4C). These results demonstrated that DATS-MSNs could alleviate hypoxia-induced inflammation in ECs.

Since mitochondrial damage is a key factor leading to an increased cellular inflammatory response, the treated ECs were subjected to JC-1 mitochondrial membrane potential assay to investigate their mitochondrial membrane integrity. As expected, the EC population treated with $3.13 \mu \mathrm{g} / \mathrm{mL}$ of DATS-MSNs showed a statistically significant increase 

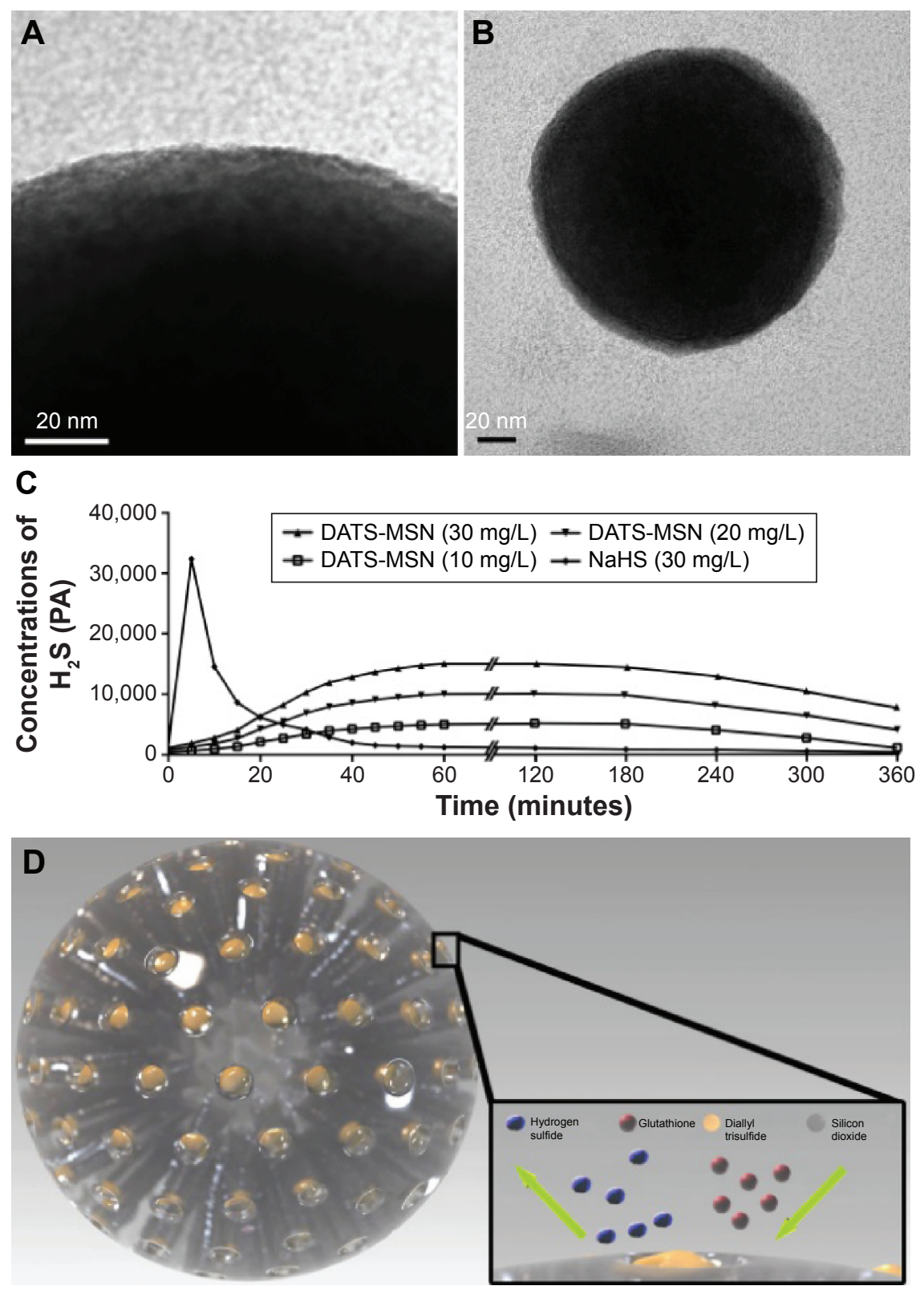

Figure I The characterization and inner workings of DATS-MSN.

Notes: TEM of the porous structure $(\mathbf{A})$ and the diameter $(\mathbf{B})$ of DATS-MSNs; controlled release of hydrogen sulfide from DATS-MSNs at concentrations of I0 mg/L, $20 \mathrm{mg} / \mathrm{L}$, and $30 \mathrm{mg} / \mathrm{L}$ in the UW solution supplemented with $2 \mathrm{mM} \mathrm{GSH}(\mathbf{C})$; and a schematic diagram of the controlled release mechanism of $\mathrm{H}_{2} \mathrm{~S}$ from DATS-MSN (D). Abbreviations: DATS-MSNs, diallyl trisulfide-loaded mesoporous silica nanoparticles; GSH, glutathione; TEM, transmission electron microscopy; UW, University of Wisconsin; PA, pico-ampere.

in the percentage of red-fluorescent cells $(58.3 \% \pm 11.2 \%$, Figure 4D) compared with the untreated control group $(37.5 \% \pm 9.3 \%$, Figure 4E; the relevant statistics are shown in Figure 4F), suggesting that the nanoparticles could effectively attenuate hypoxia-induced mitochondrial damage in ECs.

\section{DATS-MSNs inhibited apoptosis of intimal cells}

To further investigate the protective effects of DATS-MSNs on the endothelium of transplanted aortas, aortic segments were first preserved in UW solution supplemented with (Figure 5A) or without (Figure 5B) DATS-MSNs and subsequently anastomosed onto recipients' abdominal aortas before being surgically removed for examination by TUNEL assay in 3 days. As described earlier, transplanted aortas that were treated with DATS-MSNs during preservation showed negative staining along with the endothelium (Figure 5A), whereas those preserved in plain UW solution displayed clear signs of serious EC apoptosis as evidenced by the increased number of TUNEL-positive cells. Taken 

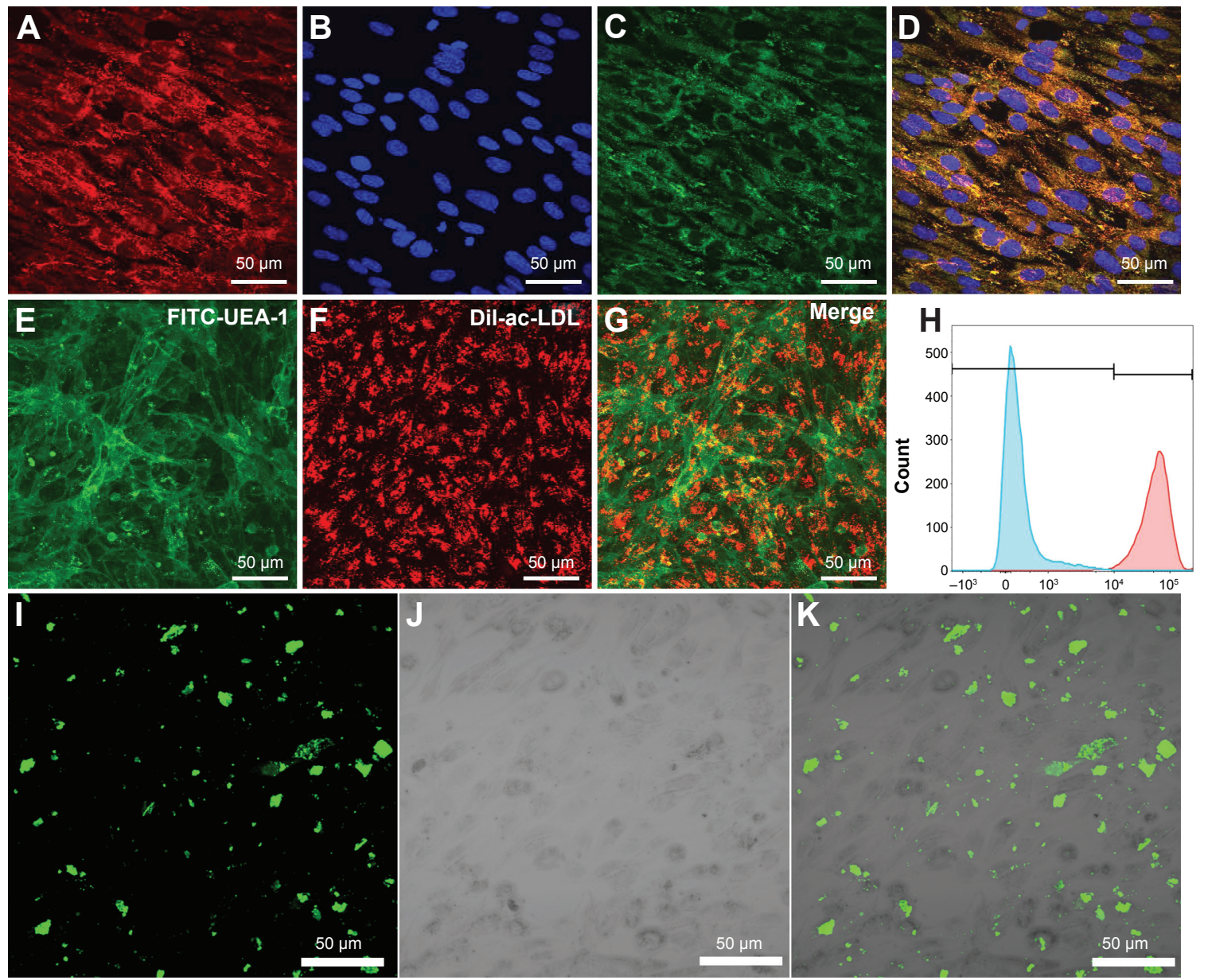

Figure 2 Confocal images and flow cytometric analysis of ECs.

Notes: $\operatorname{vWF}(\mathbf{A})$, nucleus $(\mathbf{B})$, and $C D 3 \mathrm{I}(\mathbf{C})$ of ECs were stained and then merged (D). UEA-l on endothelial cell membrane $(\mathbf{E})$ and ingested LDL $(\mathbf{F})$ were shown and then merged (G). The purity of the harvested ECs was determined by staining with PE-conjugated antihuman CD3I antibody to be $98.8 \%$ (H). FITC-conjugated DATS-MSNs (I) were located in ECs $(\mathbf{J})$ indicated by the green fluorescence in cytoplasm $(\mathbf{K})$.

Abbreviations: DATS-MSNs, diallyl trisulfide-loaded mesoporous silica nanoparticles; ECs, endothelial cells; PE, phycoerythrin; LDL, low density lipoprotein; FITC, fluorescein isothiocyanate.

together, the results confirmed that DATS-MSNs added to organ preservation solutions could effectively prevent IR-induced allograft injuries.

\section{Discussion}

Clinical application of $\mathrm{H}_{2} \mathrm{~S}$ necessitates a sustained and steady release profile. The ability of DATS-MSN to release $\mathrm{H}_{2} \mathrm{~S}$ at a stable rate for a minimum period of 4 hours makes it particularly well-suited as a delivery vehicle for use in organ preservation. Moreover, the kinetics of $\mathrm{H}_{2} \mathrm{~S}$ generation can be conveniently manipulated by adjusting the dosage of nanoparticles. The current study demonstrated that DATSMSNs, when dosed at suitable concentrations, can promote the proliferation and differentiation of ECs in vitro and alleviate inflammatory response caused by hypoxia-reoxygenation. Similar benefits were also observed in vivo, where DATSMSNs were shown to be able to protect the endothelium of aortic allografts from IR injuries.
Despite its potential as an $\mathrm{H}_{2} \mathrm{~S}$-releasing agent, clinical use of DATS is severely limited by its cytotoxicity and inability to produce $\mathrm{H}_{2} \mathrm{~S}$ in a controlled fashion. We rationalized that these shortcomings could be addressed by exploiting the highly porous architecture of MSNs to manipulate the rate at which DATS reacts with oxidizing agents. To our gratification, we found that DATS-loaded MSNs exhibited excellent biocompatibility and could maintain a stable $\mathrm{H}_{2} \mathrm{~S}$ release profile over a minimum period of 4 hours. Among the various concentrations of nanoparticles that we tested, we showed that using $3.13 \mu \mathrm{g} / \mathrm{mL}$ or $6.25 \mu \mathrm{g} / \mathrm{mL}$ of the donorloaded nanoparticles would yield the best protection results while avoiding the undesirable side effects associated with $\mathrm{H}_{2} \mathrm{~S}$ overdose. It should be noted that these dosage levels are significantly lower than what was used in a previous study ${ }^{11}$ and thus should provide greater clinical safety for patients.

Our nanoparticle-based delivery system offers several advantages. First, at an average particle size of $175 \pm 35 \mathrm{~nm}$, 

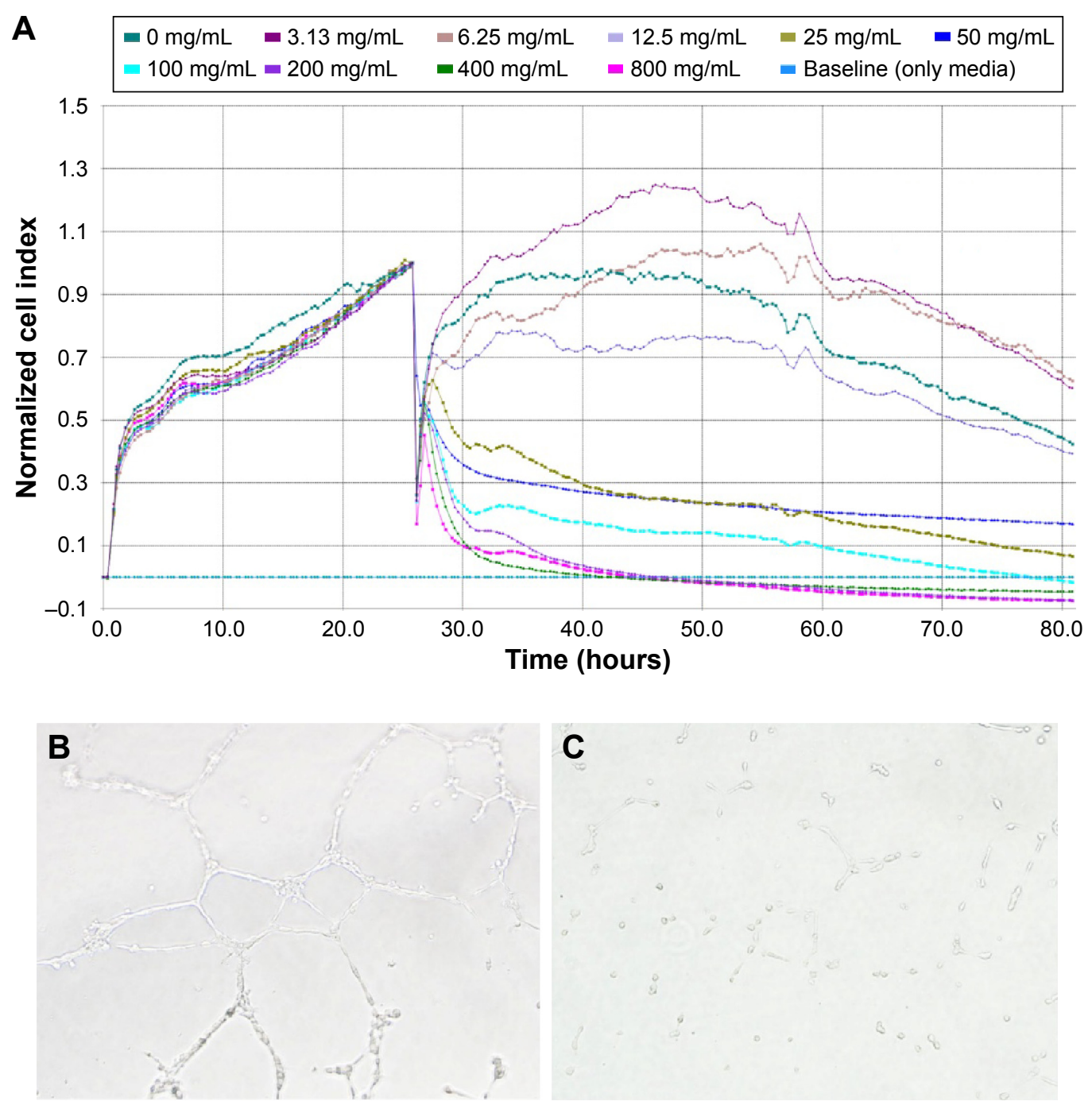

C

Figure 3 DATS-MSNs can protect ECs from hypoxia-induced injuries.

Notes: The cell indexes of ECs treated with different concentrations of DATS-MSNs (A). ECs treated with DATS-MSNs $(3.13 \mu \mathrm{g} / \mathrm{mL})$ exhibited more tube structures $(\mathbf{B})$ than the untreated control group (C).

Abbreviations: DATS-MSNs, diallyl trisulfide-loaded mesoporous silica nanoparticles; ECs, endothelial cells.

DATS-MSNs are in the submicron scale, which greatly facilitates their intracellular uptake and renal clearance. Second, the nanoparticles are constructed primarily from silica-based materials, which confer an enhanced affinity for phospholipids on cell surface, leading to higher efficiency of endocytosis. Third, the presence of endogenous GSH inside ECs, coupled with the stable release of DATS by the nanoparticles, ensures targeted delivery of $\mathrm{H}_{2} \mathrm{~S}$ to the cytoplasm in a controlled manner. Finally, the internalization of DATSMSNs by ECs would allow them to localize in the intima of the coronary arteries of the donor heart for maximum clinical benefits.

Downregulation of ICAM-1 and VCAM-1 was observed in ECs treated with DATS-MSNs. These EC phenotypic biomarkers are closely associated with immune inflammatory response and play a vital role in the development of CAV by regulating the adhesion, infiltration, and activation of leukocytes. ${ }^{12}$ It has also been suggested that the expression of ICAM-1 and VCAM-1 is related to the disturbance of mitochondrial membrane potential caused by IR. ${ }^{13}$ On the other hand, beneficial effects of $\mathrm{H}_{2} \mathrm{~S}$ on mitochondrial protection have been elucidated. For example, $\mathrm{H}_{2} \mathrm{~S}$ has been shown to alleviate IR-induced mitochondrial membrane depolarization by promoting glycogen synthase kinase $3 \beta$ phosphorylation (Ser9) and inhibiting the opening of mitochondrial permeability transition pores. ${ }^{14}$ Endogenous $\mathrm{H}_{2} \mathrm{~S}$ has also been found to be capable of regulating electron transport and oxidative phosphorylation in both isolated and cell-integrated mitochondria. ${ }^{15}$ Furthermore, $\mathrm{H}_{2} \mathrm{~S}$ pretreatment of murine microvascular ECs reduced mitochondrial DNA damage that resulted from oxidative stress. ${ }^{16}$ 
A
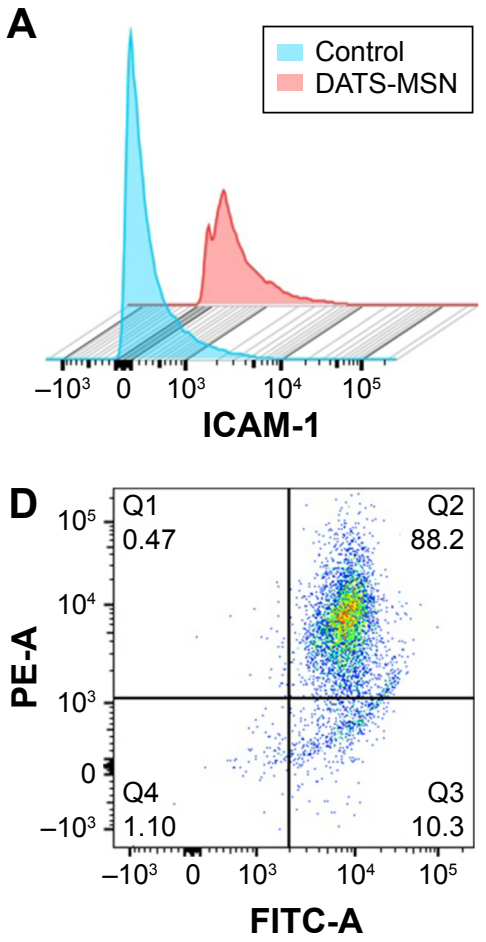

B

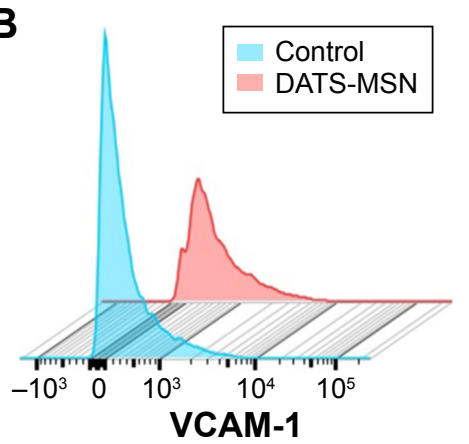

C

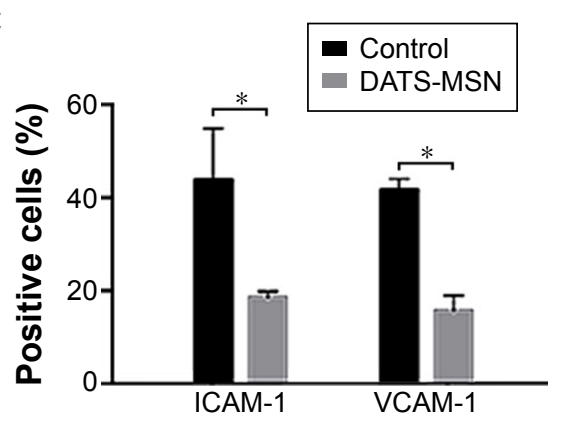

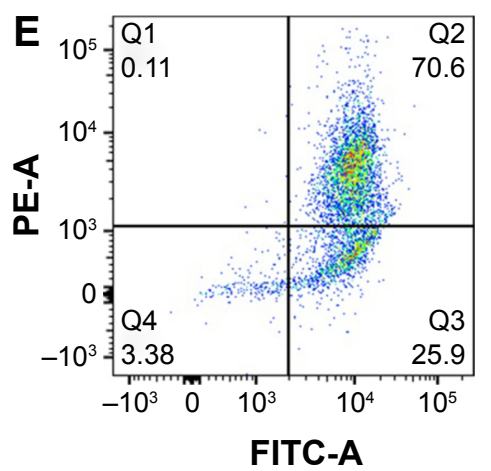

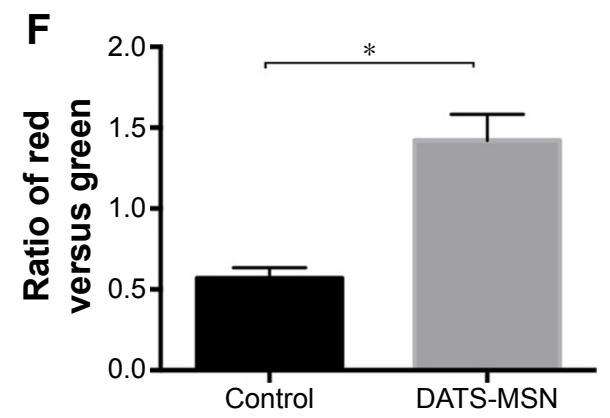

Figure 4 Flow cytometry results.

Notes: Reduced inflammatory response was characterized by decreased ICAM-I (A) and VCAM-I (B) expression levels in ECs treated with $3.13 \mu \mathrm{g} / \mathrm{mL}$ of DATS-MSNs under hypoxic conditions compared to the control group receiving no treatment. The percentages of labeled populations in both groups were also plotted in a column chart (C). DATS-MSN treatment increased the percentage of live cells (D) compared with nontreatment group (E); the difference was significant (F). Bars represent SEM. $* P<0.05$.

Abbreviations: DATS-MSNs, diallyl trisulfide-loaded mesoporous silica nanoparticles; ECs, endothelial cells; ICAM-I, intercellular adhesion molecule-I; PE, phycoerythrin; SEM, standard error of the mean; VCAM-I, vascular cell adhesion molecule-I; FITC-A, fluorescein isothiocyanate-area.

Endothelial dysfunction, such as the detachment and apoptosis of ECs, is widely regarded as a robust independent predictor of CAV. ${ }^{17}$ Our results offered convincing evidence that the proliferation and tube formation ability of ECs were substantially improved by DATS-MSN treatment.
This is consistent with a previous study suggesting that $\mathrm{H}_{2} \mathrm{~S}$ is capable of inducing angiogenesis in a dose-dependent manner. ${ }^{18}$ Exposure to $\mathrm{H}_{2} \mathrm{~S}$ has been shown to activate the ERK1/2 pathway and $\mathrm{P} 38$ pathway, ${ }^{19}$ both of which play a key role in EC proliferation and endothelial homeostasis. ${ }^{20}$
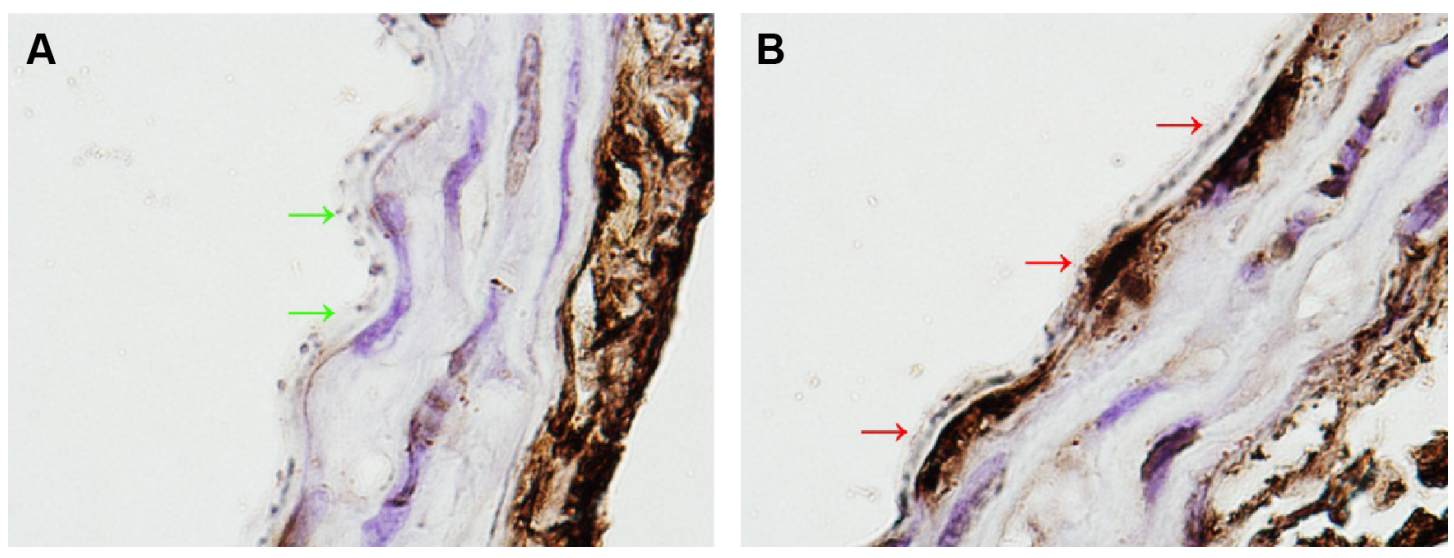

Figure 5 TUNEL results of aortic allografts.

Notes: Aortic segments were treated with preservation solution containing $3.13 \mu \mathrm{g} / \mathrm{mL}$ of DATS-MSNs (A) or containing no DATS-MSNs (B), the control group. TUNELpositive and -negative ECs were indicated by the red and green arrows, respectively.

Abbreviations: DATS-MSNs, diallyl trisulfide-loaded mesoporous silica nanoparticles; ECs, endothelial cells; TUNEL, transferase dUTP nick-end labeling. 
In addition, earlier studies suggested that $\mathrm{H}_{2} \mathrm{~S}$ could exert its protective effects by regulating the opening of $\mathrm{K}_{\mathrm{ATP}}$ channels in both cell and organelle membranes. ${ }^{21}$

\section{Conclusion}

We demonstrated that DATS-MSNs could serve as an excellent $\mathrm{H}_{2} \mathrm{~S}$ donor for ECs. Compared to classical sulfide donors such as NaHS, DATS-MSNs can generate $\mathrm{H}_{2} \mathrm{~S}$ in a sustained and steady fashion. Based on the in vitro and in vivo results, we thus envision that the application of DATS-MSN will facilitate $\mathrm{H}_{2} \mathrm{~S}$ in playing a vital role in preserving transplant organs and reducing the odds of allograft malfunction after transplantation. The subsequently prolonged preservation time implies an increased transport distance, an improved allograft function, and a sharp rise in available organ donors.

\section{Acknowledgment}

This work was supported by Natural Science Foundation of China (no 81270326).

\section{Disclosure}

The authors report no conflicts of interest in this work.

\section{References}

1. Valantine H. Cardiac allograft vasculopathy after heart transplantation: risk factors and management. J Heart Lung Transplant. 2004;23(5 suppl): S187-S193.

2. Sahara H, Sekijima M, Waki S, Ichinari Y, Shimizu A, Yamada K. Hydrogen sulfide (H2S) prevents ischemia-reperfusion injury (IRI) and prolongs survival of the fully MHC-disparate lung graft in CLAWN miniature swine. J Heart Lung Transplant. 2015;34(4):S149.

3. Koning AM, Frenay A-RS, Leuvenink HG, van Goor H. Hydrogen sulfide in renal physiology, disease and transplantation-the smell of renal protection. Nitric Oxide. 2015;46:37-49.

4. Lobb I, Davison M, Carter D, et al. Hydrogen sulfide treatment mitigates renal allograft ischemia-reperfusion injury during cold storage and improves early transplant kidney function and survival following allogeneic renal transplantation. J Urol. 2015;194(6):1806-1815.

5. Szabo C, Ransy C, Módis K, et al. Regulation of mitochondrial bioenergetic function by hydrogen sulfide. Part I. Biochemical and physiological mechanisms. Br J Pharmacol. 2014;171(8):2099-2122.

6. Whiteman M, Li L, Rose P, Tan C-H, Parkinson DB, Moore PK. The effect of hydrogen sulfide donors on lipopolysaccharide-induced formation of inflammatory mediators in macrophages. Antioxid Redox Signal. 2010;12(10):1147-1154.
7. Whiteman M, Le Trionnaire S, Chopra M, Fox B, Whatmore J. Emerging role of hydrogen sulfide in health and disease: critical appraisal of biomarkers and pharmacological tools. Clin Sci (Lond). 2011;121(11): 459-488.

8. Slowing II, Trewyn BG, Lin VS-Y. Mesoporous silica nanoparticles for intracellular delivery of membrane-impermeable proteins. $\mathrm{J} \mathrm{Am} \mathrm{Chem}$ Soc. 2007;129(28):8845-8849.

9. Tang H, Guo J, Sun Y, Chang B, Ren Q, Yang W. Facile synthesis of $\mathrm{pH}$ sensitive polymer-coated mesoporous silica nanoparticles and their application in drug delivery. Int J Pharm. 2011;421(2):388-396.

10. Chen B, Guo L, Fan C, et al. Carbon monoxide rescues heme oxygenase1-deficient mice from arterial thrombosis in allogeneic aortic transplantation. Am J Pathol. 2009;175(1):422-429.

11. Xing X, He X, Peng J, Wang K, Tan W. Uptake of silica-coated nanoparticles by HeLa cells. J Nanosci Nanotechnol. 2005;5(10):1688-1693.

12. Pober JS, Jane-Wit D, Qin L, Tellides G. Interacting mechanisms in the pathogenesis of cardiac allograft vasculopathy. Arterioscler Thromb Vasc Biol. 2014;34(8): 1609-1614.

13. van den Hoogen P, Huibers MM, Sluijter JP, de Weger RA. Cardiac allograft vasculopathy: a donor or recipient induced pathology? J Cardiovasc Transl Res. 2015;8(2):106-116.

14. Yao L-L, Huang X-W, Wang Y-G, Cao Y-X, Zhang C-C, Zhu Y-C. Hydrogen sulfide protects cardiomyocytes from hypoxia/reoxygenationinduced apoptosis by preventing GSK-3 $\beta$-dependent opening of mPTP. Am J Physiol Heart Circ Physiol. 2010;298(5):H1310-H1319.

15. Kamat PK, Kalani A, Tyagi SC, Tyagi N. Hydrogen sulfide epigenetically attenuates homocysteine-induced mitochondrial toxicity mediated through NMDA receptor in mouse brain endothelial (bEnd3) cells. J Cell Physiol. 2015;230(2):378-394.

16. Szczesny B, Módis K, Yanagi K, et al. AP39 [10-oxo-10-(4-(3-thioxo$3 \mathrm{H}-1,2$-dithiol-5yl) phenoxy) decyl] triphenylphosphonium bromide], a mitochondrially targeted hydrogen sulfide donor, stimulates cellular bioenergetics, exerts cytoprotective effects and protects against the loss of mitochondrial DNA integrity in oxidatively stressed endothelial cells in vitro. Nitric Oxide. 2014;41:120-130.

17. Singh N, Van Craeyveld E, Tjwa M, et al. Circulating apoptotic endothelial cells and apoptotic endothelial microparticles independently predict the presence of cardiac allograft vasculopathy. J Am Coll Cardiol. 2012;60(4):324-331.

18. Cai W-J, Wang M-J, Moore PK, Jin H-M, Yao T, Zhu Y-C. The novel proangiogenic effect of hydrogen sulfide is dependent on Akt phosphorylation. Cardiovasc Res. 2007;76(1):29-40.

19. Papapetropoulos A, Pyriochou A, Altaany Z, et al. Hydrogen sulfide is an endogenous stimulator of angiogenesis. Proc Natl Acad Sci U S A. 2009;106(51):21972-21977.

20. Thoppil R, Adapala R, Cappelli H, Paruchuri S, Meszaros JG, Thodeti $C$. TRPV4 channels regulate tumor angiogenesis via modulation of ERK1/2-dependent tumor endothelial cell proliferation. FASEB J. 2015;29(1 suppl):796.5.

21. Polhemus DJ, Lefer DJ. Emergence of hydrogen sulfide as an endogenous gaseous signaling molecule in cardiovascular disease. Circ Res. 2014;114(4):730-737.
International Journal of Nanomedicine

\section{Publish your work in this journal}

The International Journal of Nanomedicine is an international, peerreviewed journal focusing on the application of nanotechnology in diagnostics, therapeutics, and drug delivery systems throughout the biomedical field. This journal is indexed on PubMed Central,

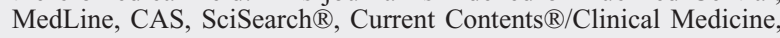

\section{Dovepress}

Journal Citation Reports/Science Edition, EMBase, Scopus and the Elsevier Bibliographic databases. The manuscript management system is completely online and includes a very quick and fair peer-review system, which is all easy to use. Visit http://www.dovepress.com/ testimonials.php to read real quotes from published authors. 